\title{
Variables cognitivas de los estudiantes universitarios: su relación con dedicación al estudio y rendimiento académico
}

\section{Cognitive Variables of University Students: their Relationship with Dedication to Study and Academic Performance}

Laura Fernanda Barrera Hernández

Javier José Vales García

Mirsha Alicia Sotelo-Castillo

Dora Yolanda Ramos-Estrada

Instituto Tecnológico de Sonora
Jesús Ocaña-Zúñiga

Universidad de Ciencias y Artes de Chiapas

\section{Resumen}

Esta investigación tuvo como objetivo probar empíricamente un modelo hipotético para explicar la dedicación al estudio y el rendimiento académico, a través de variables cognitivas de estudiantes universitarios, tales como autorregulación, orientación hacia el futuro y estrategias de aprendizaje. Participaron 187 estudiantes de una universidad del sur de México. En el modelo propuesto se predijo que la orientación hacia el futuro, autorregulación y estrategias de aprendizaje influirían en la dedicación al estudio y el rendimiento académico de los estudiantes. El modelo teórico fue probado mediante ecuaciones estructurales y los resultados mostraron indicadores de bondad de ajuste, por lo tanto, se entiende que los datos respaldan las relaciones propuestas. La relevancia de este estudio radica en su naturaleza integradora, ya que las variables en cuestión han sido estudiadas y su relación con el rendimiento académico ha sido comprobada; sin embargo, la generalidad de las investigaciones ha medido esta relación a través de correlaciones aisladas.

Palabras clave: autorregulación, orientación al futuro, estrategias de aprendizaje, dedicación al estudio, rendimiento académico.

\footnotetext{
Nota del autor

Laura Fernanda Barrera Hernández. Departamento de Psicología, Instituto Tecnológico de Sonora (ITSON). Javier José Vales García. Departamento de Psicología, Instituto Tecnológico de Sonora (ITSON). Mirsha Alicia Sotelo-Castillo. Departamento de Psicología, Instituto Tecnológico de Sonora (ITSON). Dora Yolanda Ramos-Estrada. Departamento de Psicología, Instituto Tecnológico de Sonora (ITSON). Jesús Ocaña-Zúñiga. Facultad de Ciencias Humanas y Sociales, Universidad de Ciencias y Artes de Chiapas (UNICACH). La correspondencia en relación con este artículo debe dirigirse a Laura Fernanda Barrera Hernández, Instituto Tecnológico de Sonora, 5 de Febrero 818 Sur, Col. Centro, Ciudad Obregón, Sonora, México.

Dirección electrónica: laura.barrera@itson.edu.mx
} 


\begin{abstract}
This research aimed to empirically test a model to explain the dedication to study and academic performance through cognitive variables of university students, such as self-regulation, future orientation, and learning strategies. One hundred eighty-seven students from a university in southern Mexico participated. The proposed model predicted that future orientation, self-regulation, and learning strategies would influence the dedication to study and the academic performance of students. Such model was tested through structural equations modeling; their results showed goodness of fit, so the data support the proposed relationships. The relevance lies in its integrative nature since the majority of research has measured this relationship through isolated correlations between variables.

Keywords: self-regulation, future orientation, learning strategies, study dedication, academic performance.
\end{abstract}

Una de las dimensiones más importantes en el proceso de enseñanza-aprendizaje es el rendimiento académico de los estudiantes (Ruiz, Ruiz, y Ruiz, 2010). El interés en su estudio radica en la importancia que tiene la educación en la capacidad de los alumnos para contribuir a la sociedad una vez que terminan sus estudios (Di, 2007). Encontrar buenos predictores del rendimiento es relevante, es lo principal, si lo que se busca es aportar conocimiento para que se puedan realizar intervenciones con el fin de reducir el fracaso académico o aumentar la tasa de éxito entre los estudiantes universitarios (García, Alvarado, y Jiménez, 2000).

Investigaciones previas sobre el rendimiento académico de los estudiantes universitarios permiten conocer las variables que entran en juego en términos de calidad y educación superior pública (Artunduaga, 2008; ElviraValdés, y Pujol, 2012; Ocaña, 2011; Sotelo, Echeverría, Ramos, y Barrera, 2015; Tomás-
Miquel, Expósito-Langa, y Sempere-Castelló, 2014). Asimismo, al observar su trayectoria escolar se puede identificar el comportamiento académico de los estudiantes: su rendimiento académico, aprobación de cursos, el fracaso, la repetición, demora, abandono, deserción y efectividad. La tendencia apunta directamente a la calidad de la educación y su evaluación, por ende, se considera necesario profundizar en las dimensiones, criterios, indicadores, enfoques metodológicos y estrategias de calidad en las instituciones de educación superior (Garbanzo, 2007).

Un componente indispensable para abordar el tema de la calidad de la educación superior es el rendimiento académico de los estudiantes universitarios, pues es un indicador que permite un acercamiento a la realidad educativa (Díaz, Peio, Arias, Escudero, Rodríguez, y Vidal, 2002). Estudios han destacado la asociación de diversos factores con el rendimiento académico, 
entre los que destacan: los socioeconómicos, programas de estudio, metodologías de enseñanza utilizadas, la dificultad de utilizar la enseñanza personalizada, los conceptos previos y el nivel de pensamiento formal que tienen los estudiantes (Benítez, Giménez, y Osicka, 2000). En el presente estudio se han considerado variables cognitivas de los estudiantes, como: autorregulación, orientación al futuro y las estrategias de aprendizaje.

La autorregulación del aprendizaje es un concepto que indica la supervisión y el control que los individuos poseen de sus actividades de aprendizaje, además, ayuda a evaluar el progreso en las tareas de planificación y control de las acciones necesarias para lograr el éxito en los estudios (González, Castañeda, y Maytorena, 2009). La característica más destacada atribuida a la autorregulación es que el estudiante tiene control sobre su propio aprendizaje, así como la dirección de sus procesos cognitivos y motivacionales para alcanzar sus logros académicos (Boekaerts, y Cascallar, 2006). Zimmerman (2008) señala que la autorregulación del aprendizaje representa los procesos de autodirección y autoconfianza que permiten a los estudiantes transformar sus habilidades mentales en habilidades de rendimiento académico.

Varios autores han planteado y estudiado la asociación entre la autorregulación y el rendimiento académico con resultados que apoyan las relaciones positivas y significativas entre ellos, e indicado que cuánto mayor es la autorregulación, mayor es el rendimiento académico (De la Fuente, Pichardo, Justicia, y Berbén, 2008; Elvira-Valdés, y Pujol, 2012; García-Ros, y Pérez-González, 2011; Lamas, 2008; Valle, Rodríguez, Gonzáles, Núñez-Pérez, y Rosário, 2009).

Por otra parte, la orientación temporal es una variable psicológica básica debido a que influye en todos los aspectos del comportamiento humano (Díaz-Morales, 2006) y subyace en la personalidad, la toma de decisiones y el establecimiento de objetivos (Lennings, Burns, y Cooney, 1998). Es un proceso en el que la sucesión de eventos se distribuye en clases temporales para otorgar coherencia y significado, donde los marcos de tiempo pasado, presente y futuro ayudan a codificar, almacenar y recordar eventos, metas, contingencias y contextos imaginarios (Corral, 2010). El supuesto principal de la teoría de la perspectiva del tiempo supone que el comportamiento está influenciado por la forma en que los individuos asocian su comportamiento pasado, presente y futuro (Van Beek, Berghuis, Kerkhof, y Beekman, 2011).

En este caso, la orientación temporal hacia el futuro explica que el comportamiento está determinado por la aspiración a metas y recompensas futuras; ocurre en personas que son buenas para establecer y alcanzar objetivos, planificar estrategias y cumplir obligaciones a largo plazo. Las personas con una orientación futura tienden a evitar comportamientos $y$ situaciones de riesgo, y a establecer objetivos 
futuros; son conscientes, controladas, organizadas, creativas, confiables y responsables (Zimbardo, y Boyd, 1999).

La perspectiva temporal se ha correlacionado con una gran cantidad de conceptos psicológicos; se ha estudiado en relación con la satisfacción con la vida (Martínez, 2004; Palgi, y Shmotkin; 2010, Zimbardo, y Boyd, 1999; Zuzanek, 1998), trastornos psicológicos y personalidad (Adams y Nettle, 2009; Lennings et al., 1998), conductas proambientales (Corral-Verdugo, Fraijo-Sing, y Pinheiro, 2006; Corral-Verdugo, y Pinheiro, 2004) e indicadores académicos tales como rendimiento académico, compromiso académico y aprendizaje autorregulado (Bowles, 1999; Bembenutty, y Karabenick, 2004; Brown, y Jones, 2004).

Entre los hallazgos de investigaciones en el área educativa, Zimbardo y Boyd (1999) indican que el énfasis en la orientación hacia el futuro está positivamente relacionado con el promedio de calificaciones y horas de estudio por semana en estudiantes universitarios. En esta misma línea, González, Maytorena, Lohr y Carreño (2006) encontraron efectos directos e indirectos entre la propensión al futuro y el rendimiento académico en estudiantes universitarios.

Respecto a estrategias de aprendizaje, Woolfolk (2010) indica que se refieren a los planes generales para llevar a cabo actividades o tareas de aprendizaje, e incluyen técnicas o tácticas para llevar a cabo el plan de aprendizaje.

Robbins y colaboradores (2004) señalan que el uso de estrategias de aprendizaje y las habilidades de estudios están relacionados con un mayor promedio de calificaciones y la perseverancia académica. Del mismo modo, la investigación de Sotelo, Echeverría, Ramos y Barrera (2015) sobre la relación entre los tipos de estrategias de aprendizaje y el promedio, encontraron que las estrategias de aprendizaje con una correlación significativa con el promedio de calificaciones de los estudiantes universitarios son: elaboración, revisión, constancia, pensamiento crítico, uso del tiempo y concentración.

Dado que el rendimiento académico es un indicador de efectividad y calidad educativa, en su defecto, manifestaciones de fracaso como bajo rendimiento y deserción expresan deficiencias en un sistema universitario (Artunduaga, 2008). El estudio resulta relevante en el sentido de que permite identificar otras variables, relacionadas con el desarrollo cognitivo del estudiante, que aportarán conocimiento a la predicción del objeto de estudio. Considerando lo anterior, la presente investigación tuvo como objetivo probar empíricamente un modelo que integra variables cognitivas del estudiante de autorregulación, orientación al futuro y estrategias de aprendizaje, que en estudios anteriores han demostrado estar relacionadas con la dedicación al estudio y el rendimiento académico de manera aislada. 


\section{Método}

\section{Participantes}

Participaron 187 estudiantes de la Facultad de Ciencias Humanas y Sociales, inscritos en los programas educativos de Desarrollo Humano y Psicología en una universidad del sur de México. Los participantes fueron $76.5 \%$ mujeres y $23.5 \%$ hombres, con edades comprendidas entre $18 \mathrm{y}$ 27 años, con un promedio de 20.26 años (DE $=1.60)$. El $41.7 \%$ de los estudiantes cursaba el tercer año; $31 \%$, segundo; $26.2 \%$, primer año; $y$ $1.1 \%$, el cuarto año de universidad. El muestreo fue no probabilístico, ya que no se utilizó un muestreo aleatorio (Kerlinger, y Lee, 2002).

\section{Instrumentos}

La recolección de datos se realizó mediante la aplicación de tres escalas:

- Escala de Evaluación de Autorregulación del aprendizaje de textos ARATEX-R (Núñez, Amieiro, Álvarez, García, y Dobarro, 2015), adaptada para los fines de este estudio. Los autores de esta escala reportaron una confiabilidad de $\alpha=.86$. Consta de 12 elementos con 5 opciones de respuesta que van desde nunca (1) hasta siempre (5). Algunos ítems de esta escala son: "Antes de comenzar a estudiar, me aseguro de detener a mano todo el material que pueda necesitar".

- La subescala de Futuro del Inventario de Perspectiva de Tiempo de Zimbardo (ZTPI), desarrollada por Zimbardo y Boyd (1999), mide la orientación hacia el futuro de las personas. Nueve ítems de la escala fueron administrados con 5 opciones de respuesta que van desde muy poco aplicable (1) hasta bastante aplicable (5). Algunos ejemplos de los ítems son «Soy capaz de resistir las tentaciones cuando sé que hay trabajo por hacer». Los autores reportaron una confiabilidad de $\alpha=.80$ en esta escala, en su versión original.

- Escala de Estrategias de Aprendizaje del cuestionario de estrategias motivadas para el aprendizaje (MSQL) por Pintrich, Smith, García y Mckeachie (1991), traducido al español por Sotelo (2007); la autora reportó una confiabilidad de $\alpha=.92$ en esta escala. Once ítems en escala Likert con cinco opciones de respuesta que van desde totalmente en desacuerdo (0) hasta totalmente de acuerdo (4), que miden las estrategias de aprendizaje de los estudiantes en el uso de estrategias, tales como resúmenes, analogías y paráfrasis; estrategias para seleccionar información relevante; estrategias de repetición; gestión del tiempo; solicitud de apoyo a otros, esfuerzo para lograr los objetivos establecidos; aplicación de conocimientos en la toma de decisiones; entre otras estrategias.

Finalmente, se les solicitó a los estudiantes el autoinforme de las horas semanales de dedicación al estudio (adicionales a las horas de clases) como indicador de la dedicación al estudio, y el promedio general de calificaciones acumulado hasta el momento de la recolección de datos, como indicador de su rendimiento académico. Se utilizó el promedio de calificaciones debido a que, en el enfoque del rendimiento académico, las calificaciones escolares son una de las 
variables más utilizadas por los docentes e investigadores (Edel, 2003).

\section{Procedimiento}

El instrumento se aplicó en las aulas de clases de la Facultad de Ciencias Humanas y Sociales, con la autorización previa de los profesores, coordinadores de carrera y jefes de departamento. Se solicitó la colaboración de los estudiantes y se explicó el objetivo de la investigación, además, se indicó que su participación era voluntaria; posteriormente, los participantes firmaron un formato de consentimiento informado. Después de la recolección de datos, la información fue capturada en el paquete SPSS para el análisis de los datos.

\section{Análisis de datos}

Una vez recabados los datos se exploró la normalidad a través de los índices de asimetría y curtosis; se concluyó que se distribuyen normalmente (Pardo y Ruiz, 2005).

Posteriormente, los resultados fueron analizados utilizando estadísticas univariadas (medias, desviaciones estándar y frecuencias) y se revisó la consistencia interna de las escalas calculando el alfa de Cronbach para cada una de ellas en SPSS versión 23.0. Para obtener la relación entre variables, se utilizó la prueba $r$ de Pearson.

Para analizar las relaciones directas e indirectas entre las variables, se especificó el modelo de ecuaciones estructurales (SEM) en el paquete estadístico EQS (Bentler, 2006). Se utilizaron parcelas para optimizar la estructura de medición (Little, Cunningham, Shahar, y Widaman, 2002); las escalas se dividieron en tres indicadores para cada construcción probada. Para formar las parcelas, los autores distribuyeron al azar el número total de ítems correspondientes a cada factor en los indicadores. Para este estudio, se especificaron tres factores de primer orden: (1) autorregulación, (2) orientación al futuro y (3) estrategias de aprendizaje; donde éstos formaron un factor de segundo orden llamado «variables cognitivas del estudiante».

El modelo especificado sugería que el factor de "variables cognitivas del estudiante" influiría en la dedicación al estudio, a la vez que éste influiría significativamente en el rendimiento académico.

\section{Resultados}

En autorregulación, los estudiantes obtuvieron una media de $1.61(D E=.55)$ en una escala de 0 a 4 , lo que indica que los estudiantes reportan que casi nunca o algunas veces son autorregulados. Entre las acciones y pensamientos de autorregulación más frecuentes, señalan procurar un ambiente tranquilo, animarse a sí mismos recordando que para aprender es necesario esforzarse, y el que para comprender un texto es necesario formar relaciones entre la información nueva y los conocimientos previos. En orientación hacia el futuro, la media fue de $3.48(D E=.84)$, considerando que los puntajes iban del 1 al 5, los estudiantes reportan que los indicadores de orientación hacia el futuro se asemejan a su forma de pensar o actuar. Los 
ítems con medias más altas se refirieron a que les incomoda llegar tarde a sus compromisos, se preocupan si las cosas no se hacen a tiempo, además de evaluar costos y beneficios de sus decisiones; asimismo, se observaron medias más bajas en los ítems relacionados con planificar su día por las mañanas, hacer listas de lo que debe hacerse y terminar los proyectos a tiempo debido al progreso constante, entre otros. Respecto a las estrategias de aprendizaje, la media fue de $3.74(D E=.67)$, dado que la escala iba de 1 a 5 , este puntaje sugiere que los estudiantes reportan estar de acuerdo en aplicar esas estrategias de aprendizaje. Los ítems con medias más elevadas se refirieron a estrategias como: sacar ideas importantes de lecturas y notas de clase a la hora de estudiar, en caso de confusión irse al principio y tratar de entender, determinar los conceptos que no comprenden bien y el pedir ayuda a compañeros de clase, mientras que, aquellas que reportan utilizar en menor medida son fijarse metas para dirigir las actividades, hacerse preguntas a sí mismos para corroborar que han aprendido y el aplicar ideas de las lecturas en otras actividades como lecciones y discusiones.

Tabla 1

Estadísticas univariadas y confiabildiad de las escalas utilizadas

\begin{tabular}{|c|c|c|c|c|c|}
\hline Escala/ítems & Mín. & Máx. & $\mathrm{M}$ & D.E & Alfa \\
\hline Rendimiento académico & 6.00 & 9.81 & 8.47 & .64 & \\
\hline Dedicación al estudio & 0 & 50 & 13.31 & 12.63 & \\
\hline Autorregulación & 0 & 4 & 1.61 & 0.55 & .83 \\
\hline $\begin{array}{l}\text { Mientras estoy estudiando, me animo recordándome que comprender y } \\
\text { aprender, depende de que me esfuerce lo suficiente. }\end{array}$ & 0 & 3 & 1.93 & 0.88 & \\
\hline $\begin{array}{l}\text { Antes de empezar a estudiar, me detengo a decidir las actividades y } \\
\text { estrategias que voy a realizar, planificando cómo voy a leer y estudiarlo. }\end{array}$ & 0 & 3 & 1.50 & 0.92 & \\
\hline $\begin{array}{l}\text { Antes de empezar a estudiar, si me parece inútil o poco interesante, } \\
\text { intento motivarme recordándome lo importante que es aprenderlo para } \\
\text { poder aprobar el examen y la asignatura, y así acabar el curso, la } \\
\text { carrera. }\end{array}$ & 0 & 3 & 1.49 & 0.96 & \\
\hline $\begin{array}{l}\text { Antes de empezar a estudiar, planifico el tiempo que puedo necesitar } \\
\text { dedicar a comprender y aprender el texto y cómo voy a distribuirlo entre } \\
\text { las distintas actividades que tengo que realizar. }\end{array}$ & 0 & 3 & 1.50 & 0.92 & \\
\hline $\begin{array}{l}\text { Mientras estudio, ante las dificultades que me desaniman, intento hacer } \\
\text { algo para sentirme mejor como recordarme lo bien que me sentiré } \\
\text { cuando consiga aprender acerca de esa materia. }\end{array}$ & 0 & 3 & 1.59 & 0.92 & \\
\hline $\begin{array}{l}\text { Mientras estoy estudiando, considero si mi planificación del tiempo fue } \\
\text { correcta, o si tengo que modificarla. }\end{array}$ & 0 & 3 & 1.21 & 0.95 & \\
\hline
\end{tabular}


Continuación Tabla 1...

Cuando termino el texto si no he comprendido bien me detengo a pensar cómo le hice y qué podría mejorar para comprender mejor la próxima vez.

Después de intentar estudiar, reflexiono sobre el esfuerzo que tuve que dedicarle y utilizo esta experiencia para planificar mi actividad en futuras tareas similares.

Antes de empezar a estudiar, si hay demasiado ruido $u$ otros aspectos que me impidan concentrarme, hago algo para procurarme un ambiente tranquilo y sin distracciones.

Mientras voy leyendo, intento ir relacionando las ideas más importantes para encontrar la organización general del texto.

Para comprender bien un texto, trato de unir la nueva información que me aporta con lo que ya sé sobre el tema.

Al terminar una actividad académica, me doy cuenta de las cosas que he hecho que me han funcionado y me planteo posibles cambios en la forma que haré la tarea la próxima vez.

Orientación al futuro

Pienso que las personas deberían planear su día cada mañana.

Me preocupo si las cosas no se hacen a tiempo.

Cumplir con los plazos que están por vencerse y hacer las cosas necesarias son cosas que vienen primero que la diversión.

Me incomoda llegar tarde a mis compromisos

Cumplo a tiempo mis obligaciones con mis amigos y autoridades.

Antes de tomar una decisión, evalúo costos y beneficios de esa decisión.

Termino mis proyectos a tiempo porque mantengo un constante avance de actividades de ese proyecto.

Hago listas de las cosas que tengo que hacer.

Sigo trabajando en tareas difíciles y no interesantes, si ellas me van a ayudar a avanzar.

Estrategias de aprendizaje

Trato de comprender el material de clases por medio de conexiones entre la lectura y los conceptos de las lecciones.

Cuando estudio, me voy a las lecturas o notas de las clases y trato de sacar las ideas más importantes.

Cuando estoy estudiando leo las notas de mis clases y las lecturas una y otra vez.

Cuando me confundo acerca de algo que leo en clases trato de irme al principio y entenderlo.

Cuando estudio me pongo metas para dirigir mis actividades en cada período de estudio.

Cuando estudio, trato de determinar cuáles son los conceptos que no comprendo bien.

Cuando leo para mis materias trato de relacionar el material con lo que ya he aprendido.

\begin{tabular}{|c|c|c|c|}
\hline 0 & 3 & 1.51 & 0.91 \\
\hline 0 & 3 & 1.37 & 0.96 \\
\hline 0 & 3 & 2.09 & 0.97 \\
\hline 0 & 3 & 1.80 & 0.9 \\
\hline 0 & 4 & 1.89 & 0.89 \\
\hline 0 & 3 & 1.55 & 0.92 \\
\hline 1 & 5 & 3.48 & 0.84 \\
\hline 1 & 5 & 3.18 & 1.28 \\
\hline 1 & 5 & 3.60 & 1.25 \\
\hline 1 & 5 & 3.49 & 1.21 \\
\hline 1 & 5 & 3.76 & 1.17 \\
\hline 1 & 5 & 3.58 & 1.23 \\
\hline 1 & 5 & 3.60 & 1.2 \\
\hline 1 & 5 & 3.31 & 1.15 \\
\hline 1 & 5 & 3.27 & 1.18 \\
\hline 1 & 5 & 3.52 & 0.99 \\
\hline 1 & 5 & 3.74 & 0.675 \\
\hline 1 & 5 & 3.64 & 0.95 \\
\hline 1 & 5 & 3.98 & 1.02 \\
\hline 1 & 5 & 3.78 & 1.01 \\
\hline 1 & 5 & 3.94 & 0.93 \\
\hline 1 & 5 & 3.50 & 1 \\
\hline 1 & 5 & 3.86 & 0.88 \\
\hline 1 & 5 & 3.79 & 0.99 \\
\hline
\end{tabular}


Continuación Tabla 1...

\begin{tabular}{|c|c|c|c|c|}
\hline $\begin{array}{l}\text { Cuando no logro comprender el material de un curso le pido ayuda a } \\
\text { otro compañero de la clase. }\end{array}$ & 1 & 5 & 3.86 & 0.97 \\
\hline $\begin{array}{l}\text { Me hago preguntas para estar seguro de que entiendo el material que } \\
\text { he estado estudiando en clase. }\end{array}$ & 1 & 5 & 3.62 & 0.97 \\
\hline $\begin{array}{l}\text { Trato de aplicar ideas de las lecturas en otras actividades de clase } \\
\text { como lecciones y discusiones. }\end{array}$ & 1 & 5 & 3.47 & 1.04 \\
\hline $\begin{array}{l}\text { Si me confundo al tomar nota en clase me aseguro de corregirla } \\
\text { después. }\end{array}$ & 1 & 5 & 3.79 & 1.1 \\
\hline
\end{tabular}

Las correlaciones entre las variables son presentadas en la tabla 2, destacan las relaciones de las variables congnitivas del estudiante (autorregulación, orientación al futuro y $p<.05$ ).

Tabla 2

Matriz de correlaciones entre variables

\begin{tabular}{llllll}
\hline & RA & DE & FUT & REG & EAP \\
\hline Rendimiento Académico (RA) & & & & & \\
Dedicación al Estudio (DE) & $.19^{* *}$ & & & \\
Orientación al futuro (FUT) & $.17^{*}$ & $.20^{* *}$ & & & \\
Autorregulación (REG) & $.15^{*}$ & 0.11 & $.23^{* *}$ & & \\
Estrategias de aprendizaje (EAP) & $.18^{*}$ & $.18^{* *}$ & $.29^{* *}$ & $.54^{* *}$ & \\
\hline${ }^{* *} p<.01,{ }^{*} p<.05$ & & & &
\end{tabular}

La figura 1 presenta los resultados del modelo de ecuaciones estructurales, donde los factores de primer orden emergen coherentemente de las correlaciones entre sus indicadores, tal y como lo sugieren los valores altos y significativos $(p<.05)$ de sus cargas factoriales. Además, el constructo de segundo orden "variables cognitivas del estudiante", se produjo a partir estrategias de aprendizaje) con el rendimiento académico, y con la dedicación al estudio, las cuales fueron positivas y significativas $(p<.01$, 
lo cual respalda las relaciones propuestas teóricamente. El valor de la $R^{2}$ del modelo fue .44 , lo cual indica que las variables cognitivas del estudiante explican $17 \%$ de varianza de la dedicación al estudio, además, el modelo integrado por las "variables cognitivas del estudiante" y "la dedicación al estudio", explican
44\% de varianza del rendimiento académico. Los indicadores de bondad de ajuste del modelo incluyen los valores de $X^{2}(35$ g.l. $)=42.91, p=$ $.168, B N F I=.96, B B N N F I=.98, C F I=.99$; $R M S E A=.035$, los cuales indican un ajuste de los datos al modelo propuesto en este estudio.

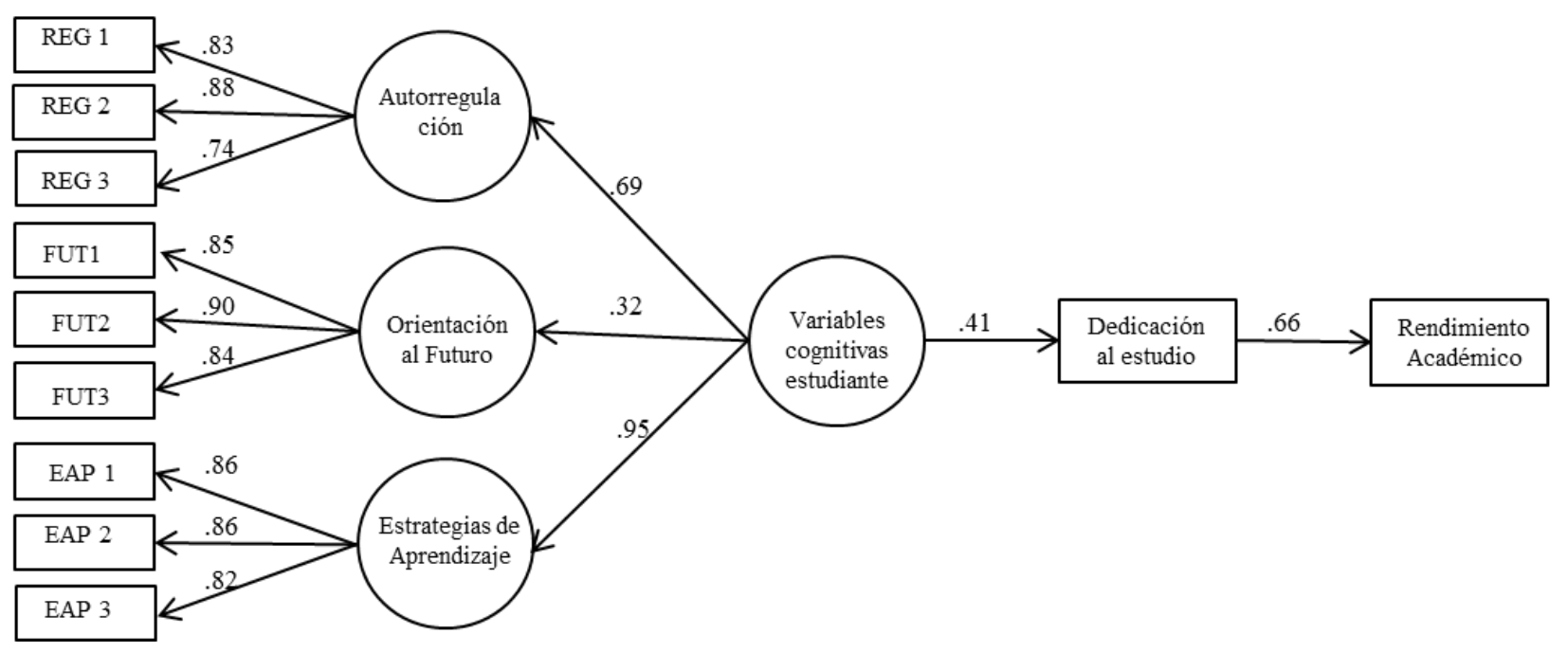

Figura 1. Modelo de dedicación al estudio y rendimiento académico a partir de variables cognitivas del estudiante. Bondad de ajuste: $X^{2}(35$ g.l. $)=42.91, p=.168, B B N F I=.96, B B N N F I=.98, C F I=.99 ; R M S E A=.035$, Horas de estudio $R^{2}=.17$, Rendimiento Académico $R^{2}=.44$

\section{Discusión}

Los resultados de la presente investigación indican que los estudiantes reportan bajos niveles de autorregulación y niveles moderados en orientación hacia el futuro y estrategias de aprendizaje. Respecto a la relación entre variables, se encontraron relaciones positivas y significativas de las variables cognitivas: autorregulación, orientación hacia el futuro y las estrategias de aprendizaje, con la dedicación al estudio y el rendimiento académico, lo cual coincide con lo informado en investigaciones previas (Elvira-Valdés, y Pujol, 2012; Sotelo et al. 2015; Zimbardo, y Boyd, 1999).

Por otra parte, el modelo de relaciones que se probó en este estudio, reveló una asociación 
significativa entre las variables cognitivas de los estudiantes, la dedicación al estudio y el rendimiento académico. De acuerdo con estos resultados, se podría asumir que los estudiantes con altos niveles de autorregulación, orientación al futuro y estrategias de aprendizaje, dedicarán más tiempo al estudio y esto impactará en su rendimiento académico.

A pesar de que los hallazgos de este estudio ratifican lo encontrado en investigaciones previas respecto a la relación entre estas variables cognitivas de los estudiantes y el rendimiento académico, aportan conocimiento respecto al papel moderador de la dedicación al estudio en esta relación.

Finalmente, se considera preciso mencionar algunas limitaciones de la investigación. En primer lugar, se encuentra la muestra, que, al ser de carácter no probabilística, además de pertenecer a una universidad y ciudad determinada, imposibilitan la generalización de los resultados. En segundo lugar, se considera como limitante el uso de autorreportes como instrumento de medición de las variables de autorregulación, orientación al futuro, estrategias de aprendizaje, horas semanales dedicadas al estudio y promedio de calificaciones, debido a que presentan desventajas frente a otros reportes tales como informes de terceras personas (p.e. profesores, amigos o familiares de los estudiantes), registros de observación de conductas, e indicadores de rendimiento obtenidos a partir de registros de la universidad. Por último, el estudio tuvo un diseño no experimental, por lo cual presenta desventajas frente a estudios experimentales, donde se podrían comprobar estas relaciones, al manipular las variables cognitivas del estudiante, mediante un programa de intervención para aumentar la autorregulación, orientación al futuro y uso de estrategias de aprendizaje, además de analizar los efectos del programa en la dedicación al estudio y promedio de calificaciones del semestre. Por consiguiente, la presente investigación brinda pautas para desarrollar estudios en el futuro que profundicen el estudio de estas variables cognitivas de los estudiantes sobre la dedicación al estudio y el rendimiento académico, utilizando otros métodos de análisis.

\section{Referencias}

Adams, J., y Nettle, D. (2009). Time perspective, personality and smoking, body mass, and physical activity: An empirical study. British Journal of Health Psychology, 14, 83-105. https://doi.org/10.1348/135910708X299664 Artunduaga, M. (2008). Variables que influyen en el rendimiento académico en la universidad (pp. 1-17). Universidad Complutense de Madrid. http://www.esc.geologia.efn.uncor. edu/wp-content/uploads/2013/05/variablesen-el-rendimiento-acadmico-universitario. pdf

Bembenutty, H., y Karabenick, S. A. (2004). Inherent association between academic delay of gratification, future time perspective, and self-regulated learning. Educational Psychology Review, 16, 35-57. https://doi. org/10.1023/B:EDPR.0000012344.34008.5c 
Benítez, M., Giménez, M., y Osicka, R. (2000). Las asignaturas pendientes y el rendimiento académico: ¿existe alguna relación?. http:// www1.unne.edu.ar/cyt/humanidades/h-009. pdf

Bentler, P. M. (2006). EQS, Structural Equations Program Manual. Encino, CA: Multivariate Software Inc.

Boekaerts, M., y Cascallar, E. (2006). How far have we moved toward the integration of theory and practice in selfregulation? Educational Psychology Review, 18, 199-210. https://doi.org/10.1007/s10648006-9013-4

Bowles, T.(1999). Focusing on time orientation to explain adolescent self-concept and academic achievement: Part II. Testing a model. Journal of Applied Health Behavior, 1, 1-8. https:// doi.org/10.1177/016235320602900302

Brown, W.T., y Jones, J. M. (2004). The substance of things hoped for: A study of the future orientation, minority status perceptions, academic engagement, and academic performance of Black high school students. Journal of Black Psychology, 30, 248-273. https://doi.org/10.1177/0095798403260727

Corral, V. (2010). Psicología de la sustentabilidad. Un análisis de lo que nos hace pro ecológicos y pro sociales. Editorial Trillas.

Corral-Verdugo, V., Fraijo-Sing, B., y Pinheiro, J. Q. (2006). Sustainable behavior and time perspective: Present, past, and future orientations and their relationship with water conservation behavior. Interamerican Journal of Psychology, 40(2), 139-147.
Corral-Verdugo, V., y Pinheiro, J. (2004). Aproximaciones al estudio de la conducta sustentable. Medio Ambiente y Comportamiento Humano, 5(1), 1-26.

De la Fuente, J., Pichardo, M. C., Justicia, F., y Berbén, A. (2008). Enfoques de aprendizaje, autorregulación y rendimiento en tres universidades europeas. Psicothema, 20(4), 705-711.

Di, L. (2007). Rendimiento académico universitario (pp. 1-33). Universidad Nacional de la Plata. http://www.aaep.org.ar/ anales/works/ works2007/digresia.pdf

Díaz, M., Peio, A., Arias, J., Escudero, T., Rodríguez, S., y Vidal, G. (2002). Evaluación del Rendimiento Académico en la Enseñanza Superior. Comparación de resultados entre alumnos procedentes de la LOGSE y del COU. Revista de Investigación Educativa, 2(20), 357-383.

Díaz-Morales, J. F. (2006). Estructura factorial y fiabilidad del Inventario de Perspectiva Temporal de Zimbardo. Psicothema, 18(3), 565-571.

Edel, R. N. (2003). El rendimiento académico: concepto, investigación y desarrollo. REICE. Revista Iberoamericana sobre Calidad, Eficacia y Cambio en Educación, 1(2), 1-16.

Elvira-Valdés, M. A., y Pujol, L. (2012). Autorregulación y rendimiento académico en la transición secundaria-universidad. Revista Latinoamericana de Ciencias Sociales, Niñez y Juventud, 10(1), 367-378.

Garbanzo, G. (2007). Factores asociados al rendimiento académico en estudiantes universitarios, una reflexión desde la 
calidad de la educación superior pública. Revista Educación, 31, 43-63. https://doi. org/10.15517/revedu.v31i1.1252

García, M., Alvarado, J., y Jiménez, A. (2000). La predicción del rendimiento académico: regresión lineal versus regresión logística. Psicothema, 12(2), 248-252.

García-Ros, R., y Pérez-González, F. (2011). Validez predictiva e incremental de las habilidades de autorregulación sobre el éxito académico en la universidad. Journal of Psychodidactics, 16(2), 231-250.

González, D. L., Castañeda, S. F., y Maytorena, M. N. (2009). Estrategias: Referidas al aprendizaje, la instrucción y la evaluación. Pearson.

González, D. L., Maytorena, M. N., Lohr, F. E., y Carreño, E. C. (2006). Influencia de la perspectiva temporal y la morosidad académica en estudiantes universitarios. Revista Colombiana de Psicología, 15(1), 15-24.

Kerlinger, F., y Lee, H. (2002). Investigación del comportamiento. Métodos de investigación en ciencias sociales. McGraw Hill.

Lamas, H. (2008). Aprendizaje autorregulado, motivación y rendimiento académico. Liberabit, 14(14), 15-20.

Lennings, C. J., Burns, A. M., y Cooney, G. (1998). Profiles of time perspective and personality: Developmental considerations. The Journal of Psychology, 132, 629-641. https:// doi.org/10.1080/00223989809599294

Little, T., Cunningham, W., Shahar, G., y Widaman, K. (2002). To parcel or not parcel: exploring the question, weighing the merits. Structural Equation Modeling, 9, 151-173. https://doi.org/10.1207/ s15328007sem0902_1

Martínez, P. (2004). Perspectiva temporal futura y satisfacción con la vida a lo largo de ciclo vital (Tesis doctoral de la Universidad de Barcelona). http://www.tdx. cat/bitstream/handle/10803/4747/pmulde1. pdf? sequence $=1$

Núñez, J. C., Amieiro, N., Álvarez, D., García, T., y Dobarro, A. (2015). Escala de Evaluación de la Autorregulación del Aprendizaje a partir de Textos (ARATEX-R). European Journal of Education and Psychology, 8, 9-22. https:// doi.org/10.1016/j.ejeps.2015.10.002

Ocaña, Y. F. (2011). Variables académicas que influyen en el rendimiento académico de los estudiantes universitarios. Investigación Educativa, 15(27), 165-180.

Palgi, Y., y Shmotkin, D. (2010). The predicament of time near the end of life: Time perspective trajectories of life satisfaction among the old-old. Aging $y$ Mental Health, 14, 577-586. https://doi. org/10.1080/13607860903483086.

Pardo, A., y Ruiz, M. (2005). Análisis de datos con SPSS 13 Base. McGraw-Hill.

Pintrich, P., Smith, A., García, T. y Mckeachie, J. (1991). A manual for the use of the Motivated Strategies for Learning Questionaire (MSLQ). Ann-Arbor: National Center for Research to Improve Postsecondary Teaching and Learning. http://eric.ed.gov/ ERICDocs/data/ericdocs 2 sql/content_ storage_01/0000019b/80/23/3c/44.pdf 
Robbins, S. B., Lauver, K., Davis, H. L., Davis, D., Langley, R., y Caristrom, A. (2004). Psychosocial and study skill factors predict college outcomes? A metaanalysis. Psychological Bulletin, 130, 261-288. https:// doi.org/10.1037/0033-2909.130.2.261

Ruiz, G., Ruiz, J., y Ruiz, E. (2010). Indicador global de rendimiento. Revista Iberoamericana de Educación, 52(4), 1-11.

Sotelo, M. (2007). Motivación, estrategias de aprendizaje y rendimiento académico en estudiantes universitarios (Tesis de Maestría). Instituto Tecnológico de Sonora.

Sotelo, M., Echeverría, S., Ramos, D., y Barrera, L. (2015). Estrategias de aprendizaje utilizadas por estudiantes universitarios $\mathrm{y}$ su relación con el rendimiento académico. En R. Pizá, S. Mortis, M. González, y B. Orduño (Comp.). Resultados de Interacción Educativa (pp. 23-35). México: ITSON.

Tomás-Miquel, J. V., Expósito-Langa, M., y Sempere-Castelló, S. (2014). Determinantes del rendimiento académico en los estudiantes de grado. Un estudio en administración $\mathrm{y}$ dirección de empresas. Revista de Investigación Educativa, 32, 379-392. http:// dx.doi.org/10.6018/rie.32.2.177581

Valle, A. A., Rodríguez, S. M., Gonzáles, R. C., Núñez-Pérez, J. C., y Rosário, P. (2009). Diferencias en rendimiento académico según los niveles de las estrategias cognitivas y de las estrategias de autorregulación. Summa Psicológica UST, 6(2), 31-42.

Van Beek, W., Berghuis, H., Kerkhof, A., y Beekman, A. (2011). Time perspective, personality and psychopathology: Zimbardo's time perspective inventory in psychiatry. Time y Society, 20, 364-374. https://doi.org/10.1177/0961463X10373960 Woolfolk, A. (2010). Psicología educativa. Aprendizaje, enseñanza y psicología educativa. El papel de la psicología educativa. Pearson.

Zimbardo, P., y Boyd, J. (1999). Putting Time in Perspective: a valid, reliable individual differences metric. Journal of Personality and Social Psychology, 66, 742-752.

Zimmerman, B. J. (2008). Investigating selfregulation and motivation: Historical background, methodological developments, and future prospects. American Educational Research Journal, 45, 166-183. https://doi. org/10.3102/0002831207312909

Zuzanek, J. (1998). Time use, time pressure, personal stress, mental health, and life satisfaction from a life cycle perspective. Journal of Occupational Science, 5, 26-39. https:// doi.org/10.1080/14427591.1998.9686432

Enviado: 04/02/2020

Revisado: $27 / 03 / 2020$

Aceptado: 1/06/2020 